\title{
The Evolution of Clinical Trials in Renal Cell Carcinoma: A Status Report for 2013-2016 from the ClinicalTrials.gov
} Website

\author{
Pooja Ghatalia $^{\mathrm{a}}$, Rebecca Koenigsberg ${ }^{\mathrm{b}}$, David Pisarcik ${ }^{\mathrm{b}}$, Elizabeth A. Handorf ${ }^{\mathrm{a}}$, \\ Daniel M. Geynisman ${ }^{\mathrm{a}}$ and Matthew Zibelman ${ }^{\mathrm{a}, *}$ \\ ${ }^{a}$ Department of Hematology/Oncology, Fox Chase Cancer Center, Philadelphia, PA, USA \\ ${ }^{\mathrm{b}}$ Philadelphia College of Osteopathic Medicine, Philadelphia, PA, USA
}

\begin{abstract}
.
Background: We previously published an analysis of clinical trials in renal cell carcinoma (RCC) using the publicly available ClinicalTrials.gov registry. Here we present a 3-year update to understand clinical research current trends in RCC compared to 2013 .

Methods: The Website's advanced search function was used to search for RCC trials. The characteristics of the trial were extracted, summarized and compared to 2013 data using Fisher's exact tests.

Results: We locked our search on May 26, 2016 with 165 trials eligible, compared with 169 trials on Sep 25, 2013. There were more phase I and I/II trials in 2016 compared to 2013 (40.8\% vs 24.9\%, $p=0.05$ ). More clinical trials in 2016 compared to 2013 used immunotherapy (IT) alone or in combination with other drugs $(24.2 \%$ vs $10.7 \%, p=0.001)$, and the use of targeted therapy alone (TT) declined $(32.9 \%$ vs $47.9 \%, p=0.005)$. TT+IT combination trials more than doubled $(6.7 \%$ vs $2.3 \%, p=0.07)$. The number of trials with treatment in (neo)adjuvant settings in 2016 and 2013 were similar $(9.7 \%$ vs $10.6 \%$, $p=0.77$ ), respectively. Compared to 2013, the number of trials with non-clear cell histology remained low $(n=10)$. Many more trials were sponsored by the pharmaceutical industry in 2016 vs $2013(41.5 \%$ vs $16.0 \%, p=<0.001)$.

Conclusion: IT-based and industry sponsored clinical trials significantly increased from 2013 to 2016 with a concomitant drop in TT only trials. The increase in industry-sponsored studies may reflect the rapid uptake of expensive IT drugs. There continues to be a paucity of (neo)adjuvant studies and for non-clear cell histologies.
\end{abstract}

Keywords: Retrospective, immunotherapy, neoadjuvant, adjuvant, kidney cancer

\section{BACKGROUND}

An estimated 63,990 Americans will be diagnosed with renal cell carcinoma (RCC) and 14,400 will die of the disease in the USA in 2017 [1]. Although

\footnotetext{
${ }^{*}$ Correspondence to: Matthew Zibelman, MD, Fox Chase Cancer Center, Temple Health, 333 Cottman Avenue, Philadelphia, PA 19111-2497, USA. Tel.: +1 215728 3889; Fax: +1 2157282629. E-mail: Matthew.Zibelman@fccc.edu.
}

$63 \%$ of RCC patients present with localized, early stage disease, about $30-40 \%$ of these patients develop metastatic disease in the future [2]. About 34\% patients have regionally advanced or metastatic disease at diagnosis [3].

Over the last 15 years, the treatment paradigm of advanced RCC has rapidly evolved [4]. This began with the development of vascular endothelial growth factor (VEGF) and mammalian target of rapamycin 
(mTOR) directed therapies and has recently moved on to checkpoint inhibitors and other immunotherapeutics. Specifically, since 2005, the US Food and Drug Administration has approved 6 VEGF receptor tyrosine kinase inhibitors (TKIs), 2 mTOR inhibitors, bevacizumab and the checkpoint inhibitor nivolumab.

Given the development of a wide variety of therapeutic options for use in patients with RCC, clinical trial landscape has been evolving as well. Additionally, increasing correlative studies to identify novel markers for both prognostic and predictive purposes has expanded areas for exploration.

In 2013 we explored the ongoing clinical trials for patients with RCC registered with ClinicalTrials.gov Web site [5]. The goal of that study was to shed light on trends in clinical trials research in RCC and identify areas where more resources might be warranted. Given the advent of immune checkpoint inhibitors, other novel immunotherapeutics and various combination therapies, we conducted a second analysis of ongoing clinical trials as of May 2016 to compare with the landscape of clinical trials in 2013. Our aim was to highlight current state of therapeutic trials in RCC, highlight the evolution of trials over 3 years and identify areas that require more research efforts.

\section{MATERIAL AND METHODS}

The data for this study were collected from the ClinicalTrials.gov website. The ClinicalTrials.gov Web site was created in accordance with the mandate of the FDA Modernization Act of 1997 [6]. This act required the creation of a clinical registry that would be accessible to public, and that would report information pertaining to federally and privately funded trials testing experimental drugs. The site provides the largest database of clinical trials in the world, including almost all interventional human trials performed in the United States and 185 countries worldwide.

The advanced search option was used with the search term, "renal cell carcinoma" OR "renal cell cancer" OR "kidney carcinoma" OR "renal neoplasm" OR "kidney neoplasm". The search was further refined to "interventional" for study type and "age $\geq 18$ ", as we had in the previously published study. The prior published study captured open studies as of Sep 24, 2013. In this study our lock out date was May 26, 2016. We excluded studies that opened after Sep 25, 2013 and closed before May 26, 2016 (Fig. 1). Basket trials with $\geq 7$ tumors with non-RCC tumor histologies, terminated studies, studies with unknown status and pediatric studies were excluded.

We extracted the following information: (1) clinical trial phase; (2) recruitment status; (3) stage; (4) treatment setting (surgery/adjuvant/neoadjuvant/ metastatic); (5) intervention (surgery/radiation/chemotherapy/targeted therapy/immunotherapy/vaccine combinations); (6) study design (e.g. single arm/ combination/parallel study); (7) name of drugs; (8) whether tissue biopsy was required; (9) histology; (10) whether biomarkers were evaluated; (11) type of sample on which biomarker testing was done; (12) randomization status; (13) control group; (14) primary outcome; (15) secondary outcome; (16) number of study sites; (17) study location; (18) study sponsor; (19) target enrollment; (20) date of trial activation and closure.

For our purposes, we defined targeted therapy according to the National Cancer Institute (NCI) definition that includes drugs or other substances that block the growth and spread of cancer by interfering with specific molecules involved in tumor growth and progression. Immunotherapy was defined as a type of biological therapy that uses substances to stimulate or suppress the immune system.

The results were compiled and analyzed independently by the first 3 authors of this report and any questions or discrepancies in data collection or coding were resolved by the last 2 authors as a separate, third party arbiter. We summarized trial characteristics using frequencies and percentages. Statistical analysis was performed to compare data collected in 2016, with previously published data collected in 2013. Fisher's exact test was used to determine whether significant difference existed between these two groups.

\section{RESULTS}

A total of 263 open trials were identified on May 26, 2016, out of which 182 trials were registered after Sep 25, 2013 and 81 trials were registered before Sep 25, 2013. We excluded trials that were closed as of May 26, $2016(n=17)$. After excluding terminated studies $(n=2)$, studies with $7+$ tumors types $(n=50)$, and non-RCC tumor types $(n=39)$, non-cancerous conditions $(n=4)$ and meta-analysis $(n=1)$, we included 165 trials open as of May 26, 2016-117 trials received after, and 48 trials received before Sep 25, 2013. This compares to the 169 trials selected in the 2013 analysis. 


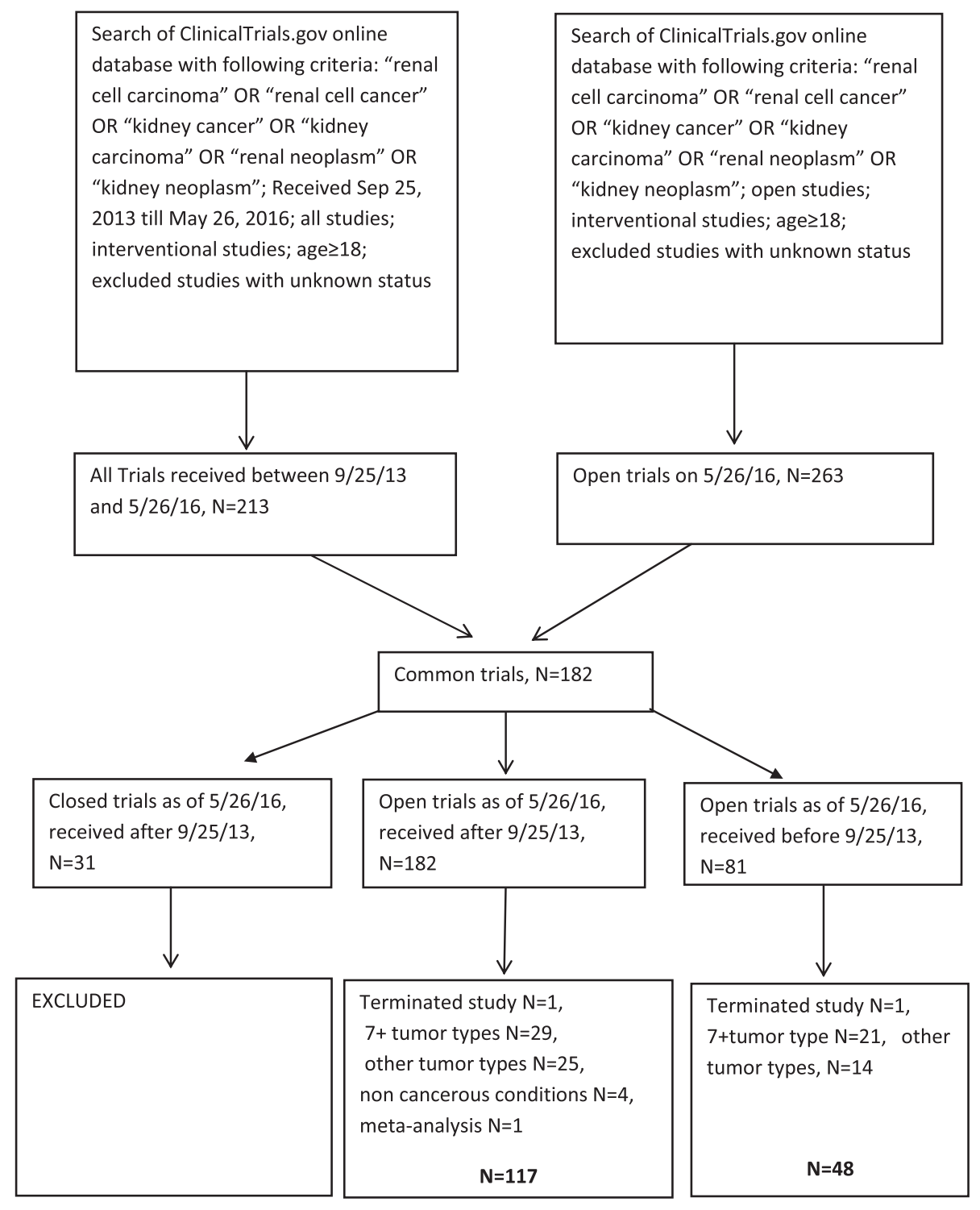

Fig. 1. Trial selection.

\section{Trial Characteristics}

Of the 165 trials included in our analysis, 132 (80.5\%) were actively recruiting, $23(14 \%)$ were not yet recruiting, $6(3.7 \%)$ were completed and 4 (2.4\%) trials did not report recruitment status. The mean target enrollment was 156 (range 4-5000) patients. There were some differences in the phases of open trials $(p=0.05)$, with the largest difference being an increased rate phase I and I/II trials in 2016 compared to 2013 (40.8\% vs $24.9 \%$ ). The proportion of phase III and IV studies was similar in the 2016 analysis compared to the 2013 analysis (11\% vs $11.8 \%$ ) (Table 1).
Trial sponsorship changed substantially $(P<0.001)$, with more trials sponsored by pharmaceutical industry in 2016 vs 2013 analysis $(41.5 \%$ vs $16.0 \%$ ). When compared to 2013 analysis, fewer trials were university ( $42 \%$ vs $53 \%$ ), cooperative group ( $2.4 \%$ vs $10 \%)$, or NCI (9\% vs $15 \%)$ sponsored. Of note, among stage I-III tumors, more trials were university sponsored than industry sponsored ( $73 \%$ vs $15.3 \%$ ) Most of the trials were conducted exclusively in the United States or Canada $(n=101$, $61.6 \%)$. Fourteen $(8.4 \%)$ multinational trials were conducted which is similar to the 2013 analysis (8.3\%). Outside of the United States, 47 (28.4\%) 
Table 1

Clinical trial characteristics of all RCC clinical trials on clinicaltrials.gov with 2013 and 2016 data

\begin{tabular}{|c|c|c|}
\hline & $\begin{array}{l}\mathrm{N}(\%) \text { in } 2013 \text { analysis } \\
\text { Total } 169 \text { trials }\end{array}$ & $\begin{array}{c}\mathrm{N}(\%) \text { in } 2016 \text { analysis } \\
\text { Total } 165 \text { trials }\end{array}$ \\
\hline \multicolumn{3}{|l|}{ Phase } \\
\hline I & $26(15.4)$ & $42(25.6)$ \\
\hline II & $67(39.6)$ & $49(29.8)$ \\
\hline III & $15(8.9)$ & $9(5.5)$ \\
\hline IV & $5(2.9)$ & $9(5.5)$ \\
\hline $\mathrm{I} / \mathrm{II}$ & $16(9.5)$ & $25(15.2)$ \\
\hline $\mathrm{II} / \mathrm{III}$ & $1(0.6)$ & $1(0.6)$ \\
\hline Unspecified/Other & $39(23.1)$ & $29(17.7)$ \\
\hline \multicolumn{3}{|l|}{ Recruitment status } \\
\hline Open & $145(86)$ & $132(80.5)$ \\
\hline Not yet open & $16(9.5)$ & $23(14)$ \\
\hline Other & $8(4.7)$ & $10(6)$ \\
\hline \multicolumn{3}{|l|}{ Sponsor } \\
\hline University & $90(53.2)$ & $69(42)$ \\
\hline Industry & $27(16)$ & $68(41.5)$ \\
\hline Cooperative group & $17(10)$ & $4(2.4)$ \\
\hline $\mathrm{NCI}$ & $25(14.8)$ & $15(9.2)$ \\
\hline Other & $10(5.9)$ & $9(5.5)$ \\
\hline \multicolumn{3}{|l|}{ Study Location } \\
\hline United States/Canada only & $102(60.4)$ & $101(61.6)$ \\
\hline Europe only & $37(21.9)$ & $14(8.5)$ \\
\hline US-based multinational & $12(7.1)$ & $13(7.8)$ \\
\hline Non-US-based multinational & $2(1.1)$ & $1(0.6)$ \\
\hline Other & $16(9.5)$ & $16(9.7)$ \\
\hline \multicolumn{3}{|l|}{ Randomized } \\
\hline Yes & $45(26.6)$ & $46(28)$ \\
\hline No & $124(73.4)$ & $119(72.6)$ \\
\hline \multicolumn{3}{|l|}{ Number of arms } \\
\hline One & $109(64.5)$ & $99(60.4)$ \\
\hline Two & $53(31.4)$ & $47(28.7)$ \\
\hline Three & $2(1.2)$ & $10(6.1)$ \\
\hline Four & $3(1.8)$ & $9(5.5)$ \\
\hline \multicolumn{3}{|l|}{ Control group } \\
\hline None & $123(72.8)$ & $120(73.1)$ \\
\hline Placebo & $11(6.5)$ & $7(4.3)$ \\
\hline Established treatment & $31(18.3)$ & $37(22.6)$ \\
\hline Supportive care & $1(0.59)$ & 0 \\
\hline Other & $3(1.8)$ & $1(0.6)$ \\
\hline
\end{tabular}

trials were conducted in Europe, $22(13.3 \%)$ in Asia, $4(2 \%)$ trials in Australia and $1(0.6 \%)$ in South/Central America. This distribution is similar to that in the 2013 analysis. While most of the trials were conducted at a single site $(n=98,59.3 \%)$, there were 21 trials that incorporated more than 10 sites. In the 2013 analysis, 30 such trials were identified. Overall, 46 (28\%) trials were randomized and $119(72.6 \%)$ trials were not.

In the 2016 analysis, 2 additional categories were recorded to capture the trial design - "combination vs single" and "parallel". The former includes trials with arms containing single agents compared with arms containing more than 1 drug. The latter includes trials with arms containing drugs with different doses that are not directly comparing outcome, as well as trials with multiple endpoints, i.e. dose determination as well as comparison with placebo. We recorded 17 $(10.3 \%)$ "combination vs single agent" trials and 19 $(11.5 \%)$ "parallel" trials.

\section{Tumor characteristics}

As in the 2013 analysis, the majority of the trials in the 2016 analysis included patients with metastatic disease $(n=99,60.4 \%)$ and a similar number of studies were performed in stage I-III disease as compared with 2013 (15.7\% vs 13.2\%). Histologically, most of the studies assessed patients with predominantly clear cell RCC $(n=57,34.7 \%)$ or all histologic subtypes 
$(n=93,56.7 \%)$. Trials specifically enrolling patients with non-clear cell tumor histology were infrequent, accounting for only 14 (8.4\%) studies. This was similar to the 2013 analysis in which 17 (10\%) studies focused on non-clear cell histologies. Papillary histology was studied in eight trials, 1 trial included chromobophobe RCC alone, three trials included any non-clear cell histology (papillary, chromophobe, sarcomatoid, medullary) and two trials included clear cell and non-clear cell histologies. Of note, most trials did not restrict the type of tumor histology (56.7\% in 2016 analysis and 57\% in 2013 analysis), and may include non-clear cell cases that were not specified. Three trials were conducted in patients with hereditary RCC (one trial in Bird Hogg Dube syndrome and two trials in hereditary leiomeiomatosis and renal cell cancer syndrome (HLRCC)).

\section{Interventions}

Among patients with metastatic tumors, 43 $(26.2 \%)$ trials included patients in any line of therapy, $26(15.8 \%)$ trials were first line metastatic, 36 $(21 \%)$ were second line or beyond and $5(3 \%)$ trials were third line or beyond. We found $10(6.1 \%)$ trials that were designed to assess interventions in the neoadjuvant setting and $4(2.4 \%)$ in the adjuvant setting. Two trials $(2.4 \%)$ used drugs in the perioperative setting (Table 2). Four of the 10 trials in the neoadjuvant setting used radiation therapy, 1 used targeted therapy, 3 used immunotherapy, 1 used a vaccine and 1 trial used high intensity focused ultrasound. In the adjuvant setting, targeted therapy was used in 3 trials and cytokine-induced killer cells were used in 1 trial, and in the perioperative setting 1 trial used immunotherapy and 1 trial used targeted therapy.

Among therapeutic interventions used, more clinical trials in 2016 compared to 2013 used immunotherapy (IT) alone or in combination with other drugs $(24.2 \%$ vs $10.7 \%, p=0.001)$, and the use of targeted therapy alone (TT) declined $(32.9 \%$ vs $47.9 \%, p=0.005)$. IT alone was used in $27(16.4 \%)$ of current trials, compared to $10(5.96 \%)$ in 2013. TT+IT combination trials more than doubled $(6.7 \%$ vs $2.3 \%, p=0.07$ ) (Table 3 ).

Among 26 trials with localized disease (stage I-III tumors), 8 trials were surgical in nature or involved a therapeutic equivalent (i.e cryoablation), 5 trials involved radiation therapy, 3 involved use of targeted therapy, 2 involved use of immunotherapy and 1 trial used a vaccine.

\section{Immunotherapy trials}

Among the 40 trials that used immunotherapy as single agent or as combination therapy, 3 trials contained either high dose interleukin-2 or interferonalfa, 30 trials involved anti PD-1/PD-L1 therapy and 11 trials used other immunotherapies (Table 4). Anti PD-1 and PD-L1 therapies have been combined with targeted therapies such as bevacizumab, pazopanib, ziv-aflibercept, lenvatinib and axitinib, as well as with other novel checkpoint inhibitors like anti T-cell immunoglobulin mucin-3 (TIM3), anti lymphocyteactivation gene 3 (LAG-3) and anti CD27. Among the immunotherapy trials, 35 trials were in the metastatic setting ( 5 first line, 12 second line or beyond, 18 any line), 3 trials were in the neoadjuvant setting and 1 trial was in the perioperative setting. One trial did not report the treatment setting.

\section{Biomarkers}

Among 82 (50\%) trials testing biomarkers (compared to $45 \%$ in 2013), 31 trials examined targeted therapy and 24 trials examined immunotherapy. This is in contrast to the 2013 analysis where biomarkers were examined in 52 targeted therapy and 9 immunotherapy trials. While 7 of $14(50 \%)$ surgical trials were randomized, $30 \%(23 / 75)$ TT trials and $25 \%(10 / 36)$ IT trials were randomized. This trend is consistent with the 2013 analysis. Among trials that included biomarker testing, $15(18 \%)$ tested tumor tissue, 37 (48.7\%) used blood, 15 (18\%) tested both blood and tissue, 11 (13.4\%) used imaging and 4 (4.8\%) used urine.

\section{DISCUSSION}

While the number of trials and the basic study characteristics in our follow-up survey remained largely unchanged from the 2013 survey, there were several interesting observations that were noted. Most notably, and not unexpectedly, there were more clinical trials in 2016 compared to 2013 using IT alone or in combination with other drugs $(24.2 \%$ vs $10.7 \%, p=0.001$ ), and the use of TT declined $(32.9 \%$ vs $47.9 \%, p=0.005)$. IT alone was used in $16.4 \%$ of current trials, compared to $5.96 \%$ in 2013. TT+IT combination trials more than doubled $(6.7 \%$ vs $2.3 \%, p=0.07)$. This reflects the evolving treatment paradigm in patients with metastatic RCC, and other metastatic cancers, in which IT 
Table 2

Clinical trial and tumor characteristics of all RCC clinical trials on clinicaltrials.gov with 2013 and 2016 data

\begin{tabular}{|c|c|c|}
\hline Characteristic & $\begin{array}{c}\mathrm{N}(\%) \text { in } 2013 \text { analysis } \\
\text { Total } 169 \text { trials }\end{array}$ & $\begin{array}{c}\mathrm{N}(\%) \text { in } 2016 \text { analysis } \\
\text { Total } 165 \text { trials }\end{array}$ \\
\hline \multicolumn{3}{|l|}{$\overline{\text { Stage }}$} \\
\hline $\mathrm{I} / \mathrm{II}$ & $18(10.6)$ & $19(11.6)$ \\
\hline $\mathrm{I} / \mathrm{II} / \mathrm{III}$ & $7(4.1)$ & $5(3)$ \\
\hline III/IV & $1(0.6)$ & $2(1.2)$ \\
\hline IV & $89(52.7)$ & $99(60.4)$ \\
\hline III-IV & $32(19)$ & $21(12.8)$ \\
\hline I-IV & $9(5.3)$ & $14(8.5)$ \\
\hline Other & $13(7.7)$ & $5(3)$ \\
\hline \multicolumn{3}{|l|}{ Histology } \\
\hline All & $97(57.4)$ & $93(56.7)$ \\
\hline Clear cell & $53(31.4)$ & $57(34.8)$ \\
\hline Non-clear cell & $17(10)$ & $14(8.5)$ \\
\hline \multicolumn{3}{|l|}{ Treatment setting } \\
\hline Surgery of equivalent & $26(15.4)$ & $22(13.4)$ \\
\hline Neoadjuvant/Adjuvant & $18(10.6)$ & $16(9.7)$ \\
\hline Metastatic any line & $33(19.5)$ & $43(26.2)$ \\
\hline Metastatic first line only & $34(20.1)$ & $26(15.9)$ \\
\hline Metastatic second line or beyond & $35(20.7)$ & $36(21.9)$ \\
\hline Metastatic third line or beyond & $5(3)$ & $5(3)$ \\
\hline Metastatic first or second line & $6(3.5)$ & $4(2.4)$ \\
\hline Other & $12(7.1)$ & $12(7.3)$ \\
\hline \multicolumn{3}{|l|}{ Intervention } \\
\hline Surgery or equivalent & $18(10.6)$ & $14(8.5)$ \\
\hline Radiation & $6(3.5)$ & $12(7.3)$ \\
\hline Targeted therapy & $81(48)$ & $53(32.1)$ \\
\hline Immunotherapy & $10(5.9)$ & $27(16.4)$ \\
\hline Vaccine & $3(1.8)$ & $3(1.8)$ \\
\hline Chemoimmunotherapy & $1(0.6)$ & $2(1.2)$ \\
\hline Antibody drug conjugate & $2(1.2)$ & $1(0.6)$ \\
\hline Chemo + targeted therapy & $5(3)$ & $1(0.6)$ \\
\hline Immuno + targeted therapy & $4(2.4)$ & $11(6.7)$ \\
\hline Imaging & $12(7.1)$ & $12(7.3)$ \\
\hline Other & $18(10.7)$ & $19(11.5)$ \\
\hline Other + targeted therapy & $7(4.1)$ & $10(6.1)$ \\
\hline \multicolumn{3}{|l|}{ Biomarkers } \\
\hline Yes & $76(45)$ & $82(50)$ \\
\hline No & $92(54.4)$ & $82(50)$ \\
\hline \multicolumn{3}{|l|}{ Primary Outcome } \\
\hline Overall survival & $5(3)$ & $5(3)$ \\
\hline Disease-free survival & $8(4.7)$ & $4(2.4)$ \\
\hline Progression-free survival & $32(19)$ & $28(17)$ \\
\hline Response rate & $18(10.7)$ & $21(12.8)$ \\
\hline Feasibility/toxicity/dose-finding & $45(26.6)$ & $60(36.5)$ \\
\hline Biomarker outcome & $9(4.3)$ & $12(7.3)$ \\
\hline Other & $49(29)$ & $23(14)$ \\
\hline \multicolumn{3}{|l|}{ Type of Trial* } \\
\hline Single & $53(41.4)$ & $89(54)$ \\
\hline Combined & $28(21.8)$ & $18(10.9)$ \\
\hline Versus & $6(4.7)$ & $21(12.7)$ \\
\hline Sequential & $5(3.9)$ & $1(0.5)$ \\
\hline Alternating & $4(3.1)$ & 0 \\
\hline Combination vs single** & NR & $17(10.3)$ \\
\hline Parallel $^{* *}$ & NR & $19(11.5)$ \\
\hline
\end{tabular}

*Only 128 trials in the 2013 analysis reported the "type of trial". ${ }^{* *}$ These categories were not included in the 2013 analysis.

drugs targeting the PD-1 pathway are being explored alone and in combination in various settings to maximize the efficacy of these exciting agents. We predicted this would be the case in our 2013 discussion and the current results confirm, and maybe even exceeded our expectations from that time [5]. 
Table 3

Trials using targeted therapy and immunotherapy combinations

\begin{tabular}{lcc}
\hline Characteristic & $\begin{array}{c}\mathrm{N}(\%) \text { in 2013 } \\
\text { analysis Total } \\
169 \text { trials }\end{array}$ & $\begin{array}{c}\mathrm{N}(\%) \text { in 2016 } \\
\text { analysis Total } \\
165 \text { trials }\end{array}$ \\
\hline TT with immunotherapy & $4(2.4)$ & $10(5.9)$ \\
TT with chemotherapy & $5(3)$ & $1(0.6)$ \\
TT with other therapy & $7(4.1)$ & $11(6.6)$ \\
IT with chemotherapy & $1(0.6)$ & $2(1.2)$ \\
\hline
\end{tabular}

*These categories were created for coding in the 2016 analysis but was not used in the 2013 analysis.

We anticipate the focus on IT-based trials to continue to grow. One note of caution that has been expressed in both the medical and lay literature is in regards to the rational consideration of our most valuable resource used in all of these trials-our patients [7, 8]. As more and more IT-based trials come to fruition, less patients are available to populate them, creating a potential investigational deficit that could limit clinical progress. For example, combination of anti-PD1/PDL1 therapy with anti-VEGF antibody was used in 4 trials (nivolumab+bevacizumab, pembrolizumab+ ziv-aflibercept and two trials atezolizumab+ bevacizumab). Similarly, anti-PD1/PDL1 therapy with VEGFR TKIs were used in at least four trials and pembrolizumab+SBRT was used in two trials (Table 5). Investigators must be cognizant of this imbalance, and we as a community must strive to ensure the focus is on rational and novel combinations, concepts, and designs that may help answer important questions that will lead to better patient outcomes, and not duplicative trials that would only result in the approval of similar, and very expensive drugs. For example, it is encouraging to see other trials studying novel checkpoint inhibitors like anti CSF1R, IL-15 agonist, anti LAG3, anti CD27 and anti TIM03, and other agents like HDAC and glutaminase inhibitors. These agents are not currently used in clinical practice, and they may offer a potentially new option to patients who have exhausted standard frontline options. How trials are approved and funded may well dictate the application of these concepts.

Accordingly, we recorded a statistically significant increase in industry sponsored trials in our 2016 analysis compared to 2013. The reasons for this are likely multifactorial, but may on some level reflect the changing landscape of available funding mechanisms for oncology trials. One hypothesis to explain this would be a decrease in the amount of funding to support oncology research being dispersed by the National Cancer Institute (NCI), which is the predominant source of public funding for clinical oncology trials. However, this appears not to be the case, as publicly available reported expenses published on the NCI website [9] demonstrates slight increases in funding overall on an annual basis between 2013-2016, and this includes when broken down by funding mechanism and when considering a cumulative inflation rate of $3 \%$ over that time period. This was not looked at specifically for spending on trials focused on RCC, which may still account for part of the discrepancy. Another potential reason may be related to the increasingly expensive novel immunotherapy agents that are being tested in RCC trials today and as shown have increased in research focus. Also, given the intense competition that is inherent in trying to get similar drugs to market, pharmaceutical companies may be more willing to undertake the expense of a registration trial or large earlier stage trial in order to ensure control for eventual submission to regulatory agencies. This changing paradigm deserves monitoring and if persistent, may be ripe for further research to better determine the underlying reasons. While pharmaceutical-sponsored trials remain a necessity to support the breadth of research still needed for RCC, a dramatic shift of funding to predominantly industry-sponsored trials may have implications for the types of trials performed, favoring established drugs and combinations with existing infrastructure, and marginalizing novel concepts and compounds.

While our 2013 analysis did not capture the categories "combination vs single agent" trials and "parallel" trials, we believe that the necessity to add these designations reflects that more trials are using novel designs. The parallel trials included trials such as the "NivoPlus" study containing several arms with nivolumab combination therapies. This reflects the increasing trend to conduct large scale, early-phase trials that not only explore dose or single agent efficacy, but are designed to perform comparisons with existing standard therapies in an effort to expedite the path to approval. One contemporary example of this in the RCC space might be the trial that led to the approval of the combination of lenvatinib/everolimus for metastatic RCC [10]. This 3 arm, phase II trial compared lenvatinib to everolimus or the combination for patients with metastatic RCC refractory to one TKI. Although it included only about 50 patients per arm, it met its primary endpoint by demonstrating a PFS benefit for the combination compared to everolimus alone leading to FDA approval. This underscores the evolving perspective of the US FDA 
Table 4

Immunotherapeutic agents used in trials in 2016 analysis

\begin{tabular}{|c|c|c|c|}
\hline Immunotherapy agent & $\begin{array}{c}\text { Number of } \\
\text { trials }\end{array}$ & Phase & Treatment setting \\
\hline \multicolumn{4}{|l|}{ Cytokines } \\
\hline Aldesleukin + Entinostat (HDAC inhibitor) & 1 & $1 / 2$ & Metastatic any line \\
\hline High dose IL2 + SBRT & 1 & 2 & Metastatic any line \\
\hline rSIFN-co (super compound interferon) & 1 & 1 & Metastatic any line \\
\hline \multicolumn{4}{|l|}{ Anti PD-1 } \\
\hline \multirow[t]{4}{*}{ Nivolumab } & 4 & 1 & Neoadjuvant \\
\hline & & 1 & Neoadjuvant \\
\hline & & 4 & Metastatic 2nd line or beyond \\
\hline & & 2 & Metastatic first line \\
\hline Nivolumab +/- Bevacizumab or Ipilimumab & 1 & 1 & Metastatic any line \\
\hline Nivolumab + HBI 8000 (HDAC inhibitor) & 1 & $1 / 2$ & Metastatic any line \\
\hline Nivolumab + SABR & 1 & $1 / 2$ & Metastatic second line or beyond \\
\hline Nivolumab + CB-839 (glutaminase inhibitor) & 1 & $1 / 2$ & Metastatic any line \\
\hline Nivolumab + chemotherapy & 1 & 2 & Metastatic any line \\
\hline Nivolumab + FPA008 (anti CSF1R) & 1 & 1 & Metastatic any line \\
\hline Pembrolizumab & 1 & 1 & Neoadjuvant \\
\hline \multirow[t]{2}{*}{ Pembrolizumab + SBRT } & 2 & 2 & Metastatic second line or beyond \\
\hline & & 1 & Metastatic any line \\
\hline Pembrolizumab + pazopanib & 1 & 1 & Metastatic second line or beyond \\
\hline Pembrolizumab + ziv-aflibercept & 1 & 1 & Metastatic second line or beyond \\
\hline Pembrolizumab + vorinostat (HDAC inhibitor) & 1 & 1 & Metastatic any line \\
\hline Pembrolizumab + lenvatinib & 1 & $1 / 2$ & Metastatic any line \\
\hline PDR001 (anti PD1) + NIZ985 (IL-15 agonist) & 1 & 1 & Metastatic second line and beyond \\
\hline LAG525 (anti LAG-3) +/- PDR001 (anti PD1) & 1 & $1 / 2$ & Metastatic any line \\
\hline MBG453 (anti TIM03) +/- PDR001 (anti PD1) & 1 & $1 / 2$ & Metastatic any line \\
\hline \multicolumn{4}{|l|}{ Anti PD-L1 } \\
\hline Avelumab & 1 & 1 & Metastatic second line or beyond \\
\hline Avelumab + axitinib vs sunitinib & 1 & 3 & Metastatic first line \\
\hline Avelumab + axitinib & 1 & 1 & Metastatic first line \\
\hline Atezolizumab + bevacizumab & 1 & 2 & Metastatic any line \\
\hline Atezolizumab + bevacizumab vs sunitinib & 1 & 3 & Metastatic first line \\
\hline Atezolizumab + varlilumab (anti CD27) & 1 & $1 / 2$ & Metastatic second line or beyond \\
\hline CPI-444 (anti adenosine A2A receptor) $+/-$ atezolizumab & 1 & 1 & Metastatic second line or beyond \\
\hline Tremelimumab +/- cryoablation & 1 & 1 & Metastatic any line \\
\hline Durvalumab +/- Tremelimumab & 1 & 1 & Neoadjuvant \\
\hline Durvalumab + Tremelimumab & 1 & 1 & Metastatic any line \\
\hline \multicolumn{4}{|l|}{ Others } \\
\hline Autologous dendritic cells & 1 & 1 & Metastatic any line \\
\hline Intuvax (dendritic cell vaccine)-> sunitinib vs sunitinib & 1 & 2 & Metastatic first line \\
\hline Cytokine induced killer cells & 1 & 1 & Adjuvant \\
\hline Hyperacute Renal therapy & 1 & 1 & Metastatic any line \\
\hline APN401 (anti Cbl-b) & 1 & 1 & Metastatic second line or beyond \\
\hline Immunicel (autologous T lymphocyte therapy) & 1 & $2 / 3$ & Metastatic second line or beyond \\
\hline Dendritic cell vaccine therapy + cytokine-induced killer cell & 1 & 2 & Not reported \\
\hline Utomilumab (CD137 agonist) + /- rituximab & 1 & 1 & Metastatic any line \\
\hline Varlilumab (anti-CD27) + sunitinib & 1 & $1 / 2$ & Metastatic second line or beyond \\
\hline X4P-001 (anti CXCR4) +/- axitinib & 1 & $1 / 2$ & Metastatic second line or beyond \\
\hline INCAGN01876 (anti GITR) & 1 & $1 / 2$ & Metastatic any line \\
\hline
\end{tabular}

CSF1R: colony stimulating factor-1 receptor; GITR: Glucocorticoid induced TNF receptor; SABR: stereotactic ablative radiation therapy; SBRT: stereotactic body radiation therapy.

in how it evaluates drugs for regulatory approval, and encourages trial designs that can simultaneously evaluate multiple treatment modalities or endpoints in a single study. Whether this departure from the longstanding clinical trial dogma ultimately leads to more regulatory approvals and improves patient outcomes remains to be seen.
As multiple trials (ASSURE, S-TRAC, PROTECT) have failed to report an overall survival benefit of adding VEGFR TKIs in the adjuvant setting [11-13], there is a need for more trials in this perioperative space, with the goal of reducing recurrence and increasing survival in early stage kidney cancers post-nephrectomy. Surprisingly the number 
of trials conducted in the adjuvant/neoadjuvant setting did not differ between the two analyses $(9.7 \%$ vs $10.6 \%$ ). Of note, our analysis did not capture two main ongoing clinical trials conducted in the neoadjuvant/adjuvant setting - the PROSPER trial studying perioperative nivolumab and the IMmotion010 trial studying atezolizumab in adjuvant setting in high risk RCC patients.

Additionally, more studies need to be conducted in non-clear cell histologies as these tumors do not respond to the standard treatments approved for ccRCC. The number of trials including non-clear cell histologies did not differ in the two analyses $(8.5 \%$ vs $10 \%)$. Most of these studies included papillary cancers (8 of 14), 1 study included chromophobe histology and 3 studies included all non-clear cell histologies.

Our study is limited by several factors. The accuracy of data reported in ClinicalTrials.gov may not be uniform across studies. As this is a U.S. based registry, a comprehensive database of all RCC trials may not be available. Separate individuals captured data in the 2013 and 2016 analysis, and the interpretation of the definition of individual characteristics may be variable. While the data in our analysis was collected by three individuals and reviewed again by a single individual, human error in data collection is a potential source of inaccuracy. Our study included 48 trials that were also present in the 2013 analysis as these trials opened before September 24, 2013 but were also open in 2016. Additionally, 31 trials that opened after September 24, 2013 were also excluded as they closed before our lock date of May 26, 2016. However, these inclusions and exclusions were made as the goal of our study was to study the landscape of all RCC trials registered as of May 26, 2016. The strength of this study is our ability to compare the spectrum of registered clinical trials across two separate time points.

Overall, our findings reflect the current changing paradigm of systemic treatment in metastatic RCC. While more immunotherapy trials are being developed in this setting, we as a community must be cautious and cognizant to continually design trials that explore new ideas and answer new questions, and not duplicate efforts by repeating trials with similar drugs without a net benefit to the patient community. There is a clear need to develop more of these trials in the adjuvant and neoadjuvant setting to see if incremental benefit can be acquired by trying immunotherapy in patients with early stage RCC. Additionally, more trials need to be conducted specifically in the perioperative space and in patients with non clear-cell histology, as they do not respond well to standard therapies for clear cell RCC.

\section{REFERENCES}

[1] Siegel RL, Miller KD, Jemal A. Cancer Statistics, 2017. CA Cancer J Clin 2017;67(1):7-30.

[2] Dabestani S, et al., Renal cell carcinoma recurrences and metastases in primary non-metastatic patients: A population-based study. World J Urol 2016;34(8):1081-6.

[3] Surveillance, Epidemiology, and End Results Program. SEER stat fact sheets: Kidney and renal pelvis cancer. Bethesda, MD: National Cancer Institute Available from: http://seer.cancer.gov/statfacts/html/kidrp.html

[4] Choueiri TK, Motzer RJ. Systemic therapy for metastatic renal-cell carcinoma. N Engl J Med 2017;376(4):354-66.

[5] Zibelman $\mathrm{M}$, et al., A review of interventional clinical trials in renal cell carcinoma: A status report from the ClinicalTrials.gov WebSite. Clin Genitourin Cancer 2015;13(2):142-9.

[6] United States National Institutes of Health ClinicalTrials.gov Last accessed Aug 21 2017. ClinicalTrials.gov. Aug 21, 2017]; Available from: https://clinicaltrials.gov/ct2/ about-site/background

[7] New York Times, A Cancer Conundrum: Too Many Drug Trials, Too Few Patients. August 12 2017. https://www. nytimes.com/2017/08/12/health/cancer-drug-trials-encoun ter-a-problem-too-few-patients.html

[8] Chen DS, Mellman I. Elements of cancer immunity and the cancer-immune set point. Nature 2017;541(7637):321-30.

[9] Cancer.gov. https://www.cancer.gov/about-nci/budget/factbook/historical-trends/funding; accessed august 23, 2017.

[10] Motzer RJ, et al., Lenvatinib, everolimus, and the combination in patients with metastatic renal cell carcinoma: A randomised, phase 2, open-label, multicentre trial. Lancet Oncol 2015;16(15):1473-82.

[11] Ravaud A, et al., Adjuvant sunitinib in high-risk renalcell carcinoma after nephrectomy. N Engl J Med 2016;375(23):2246-54.

[12] Haas NB, et al., Adjuvant sunitinib or sorafenib for highrisk, non-metastatic renal-cell carcinoma (ECOG-ACRIN E2805): A double-blind, placebo-controlled, randomised, phase 3 trial. Lancet 2016;387(10032):2008-16.

[13] Sternberg CN, et al., Pazopanib in locally advanced or metastatic renal cell carcinoma: Results of a randomized phase III trial. J Clin Oncol 2010;28(6):1061-8. 